\title{
Search for the tritium nuclei in Earth re-entrant albedo radiation with the PAMELA experiment
}

\section{S.A. Koldobskiy*, V.V. Mikhailov}

National Research Nuclear University MEPhI (Moscow Engineering Physics Institute),

Kashirskoe highway 31, Moscow, 115409, Russia

E-mail: sakoldobskiy@mephi.ru

First results of tritium nuclei search in the cosmic ray albedo radiation with the PAMELA experiment are presented. Tritium is a light unstable nucleus with the half-life time of 12.32 years. This short lifetime in comparison with lifetime of galactic cosmic rays ( $10^{6}$ years) is the main reason why tritium can not be found in galactic cosmic rays. However some amount of the albedo tritium nuclei can be generated in the Earth atmosphere as a result of interaction of high energy galactic cosmic rays with atmosphere nuclei. Therefore these tritium nuclei can be registered in albedo fluxes measured with satellite experiment PAMELA. The first results of this high-energy tritium search are presented in this work.

35th International Cosmic Ray Conference - ICRC2017

10-20 July, 2017

Bexco, Busan, Korea

${ }^{*}$ Speaker. 


\section{Introduction}

The galactic cosmic ray tritium nuclei fluxes are of secondary origin only and can be generated as a result of the cosmic ray interactions with atoms of the interstellar medium. As the tritium nuclei are unstable (half-life of 12.3 years), the cosmic ray lifetime in the Galaxy is about $6 \times 10^{6}$ years and the atom density of the interstellar medium is very small, of the order of 1000 atoms per cm${ }^{-3}$, so the flux of such generated tritium nuclei in the Galaxy is negligible and possible can not be registered in cosmic ray experiments.

If one considers the albedo fluxes of cosmic rays, the generation of tritium nuclei by the interaction of high energy cosmic rays with atoms of the upper atmosphere could lead to the existence of their albedo fluxes. These fluxes were detected already by some number of experiments [1-4]. This paper is dedicated to development of identification method for tritium nuclei with energies from 40 to $400 \mathrm{MeV} / \mathrm{n}$ in the PAMELA experiment.

\section{The PAMELA experiment}

PAMELA (Payload for Matter-Antimatter and Light-nuclei Astrophysics) is an satellite-borne experiment carried on by Russian satellite Resurs DK-1, that was launched from Baikonur spaceport on June 15, 2006. The PAMELA experiment [5] is being conducted by a group of scientists

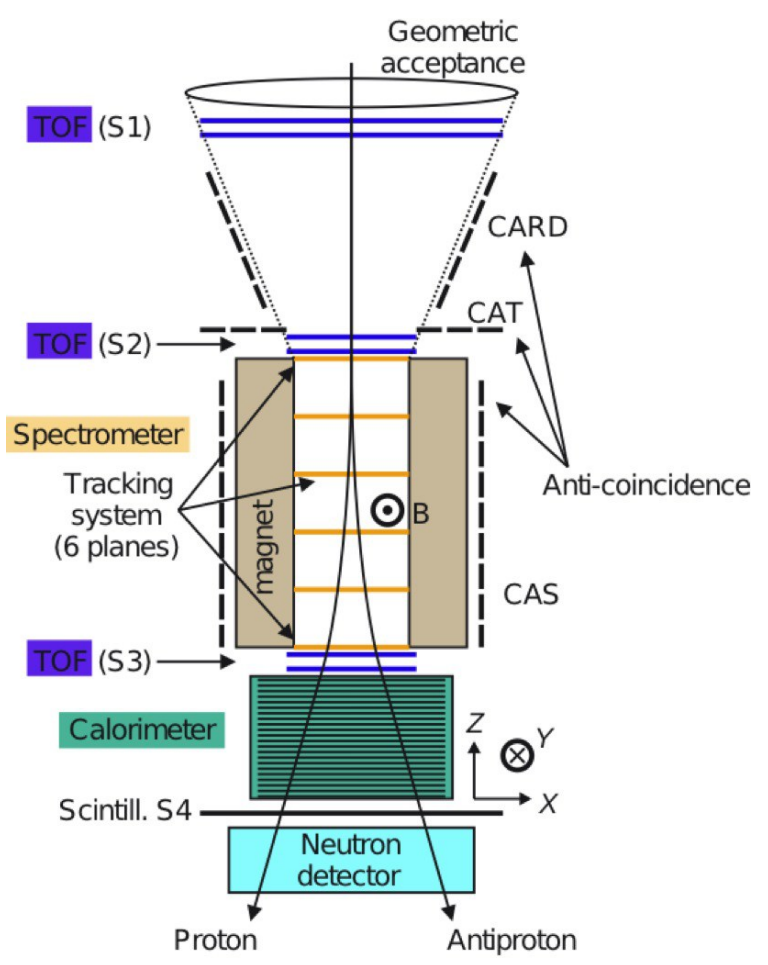

Figure 1: Scheme of PAMELA instrument.

from Russia, Italy, Germany, and Sweden. The key PAMELA instrument is the magnetic spectrometer (an arrangement of track semiconductor detectors located within the working volume of a 
permanent magnet). The unit is also fitted with a time-of-flight system that contains three doublelayer scintillation detectors, an electromagnetic calorimeter, a scintillation shower detector, and a neutron detector (Fig. 1). The working volume of the magnetic spectrometer is surrounded with scintillation counters operating in the anticoincidence (AC) mode. The AC system is used to reject events in which particles enter the spectrometer outside of its aperture.

PAMELA experimental data opened the new era in cosmic ray experiments thanks to 10-year statistics of experiment and to excellent set of high-precision detectors that allowed to identify different cosmic ray species in wide energy interval.

\section{The method of tritium nuclei identification}

The set of events satisfactory for identification procedure conditions was selected out of the entire set of information recorded in the experiment. This procedure is based on measurements of the magnetic rigidity, velocity and multiple values of energy losses. So the events with correct mentioned above measured values were selected by basic selection criteria. This criteria of "good" events selection are described in detail in work of Danil'chenko et al. [6]. After the mentioned "basic" selection the identification of nuclei was implemented by analysing of the diagram constructed in coordinates of energy losses in the tracker and rigidity (Fig. 2). One can find the groups of points

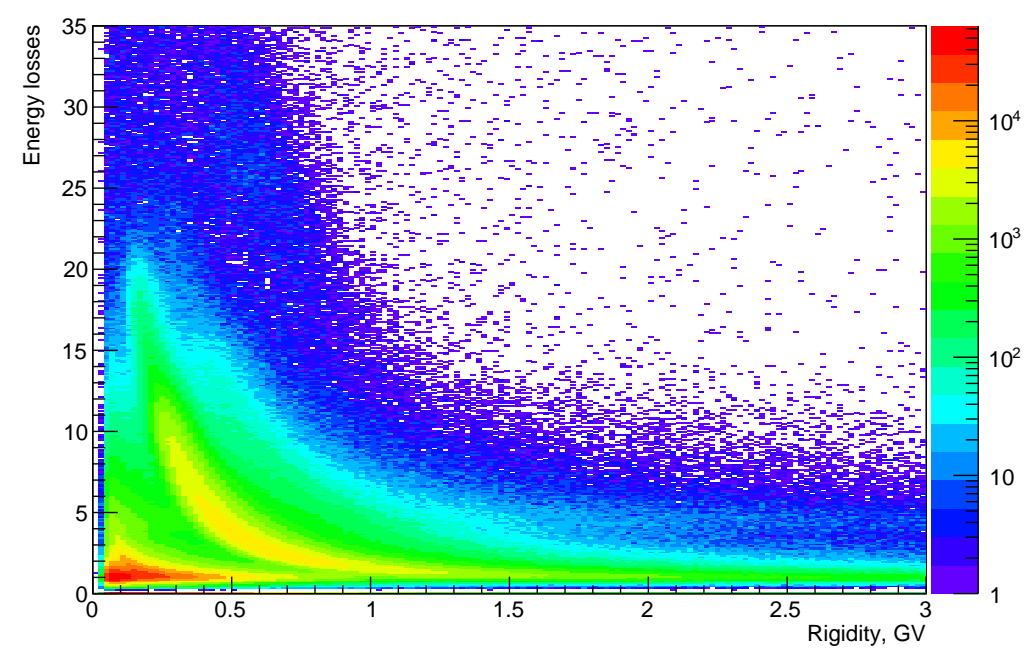

Figure 2: Energy losses in the tracker detector Y1 versus magnetic rigidity less than cutoff rigidity.

corresponding to different particle species, in particular pions, protons, deuterons. Tritium nuclei and possible some amount of $\mathrm{He} 3$ are visible also. But clear identifying of the boundaries corresponding to the different populations is difficult due to limited energy resolution. For example, tritium area is overlapped with areas of protons, deuterons and He3.

To correct identification of tritium nuclei method of background suppressing of these particles need to be used.

The method developed to suppress the background of $\mathrm{p}, \mathrm{d}, \mathrm{t}$ and He nuclei is based on multi parametric analysis of data, it described in details in the work concerned deuteron identification [6]. 
The area of possible tritium nuclei energy release in tracker was selected by two boundary energy release values for each narrow rigidity bands $\Delta R=0.1 \mathrm{GV}$ for all tracker detectors with sufficient statistics. The boundary values were chosen to minimize the losses of registered tritium nuclei and to suppress the noise of the other particle (mostly, deuterons and He3) in maximum. Then the new set of events with mean energy losses in tracker confined between the boundaries chosen in previous step was considered. The new distributions were built for mass to charge squared. Example of this picture can be found on the Fig 3.

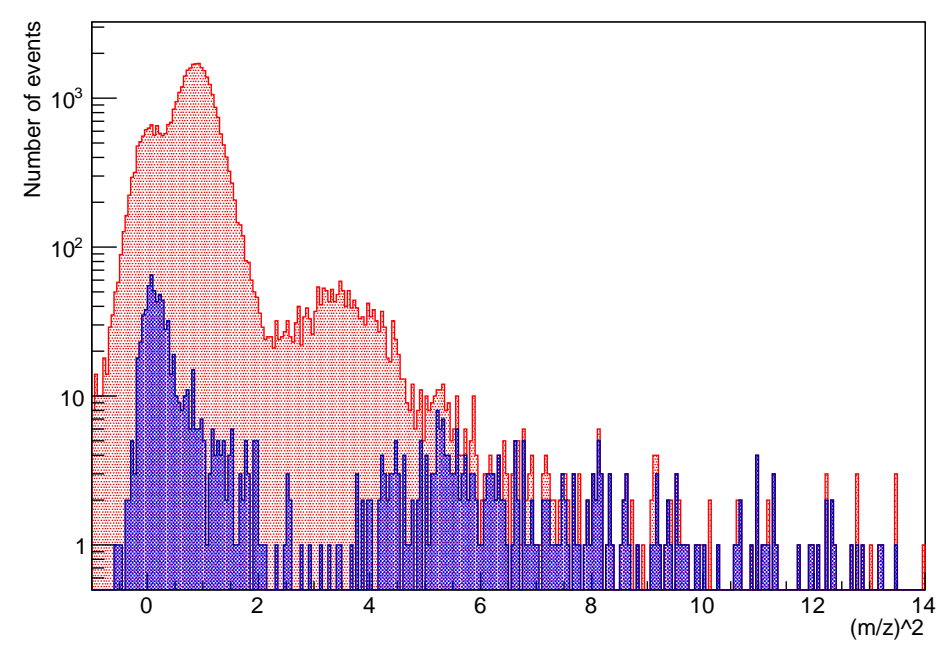

Figure 3: Mass to charge squared distribution for tritium nuclei with energies from 79.5 to 100 $\mathrm{MeV} /$ nucleon. Red distribution correspond to the dataset with "basic" selection, blue one correspond to selection of tritium. Tritium peak $\left(5.5 \mathrm{GeV}^{2}\right)$ is slightly shifted from original position $\left(7.2 \mathrm{GeV}^{2}\right)$ due to slowdown effect.

One can see that the peak of tritium nuclei are clearly watched (in the blue distribution) as well as the vast suppressed background of other events. Peak is slightly shifted from original position due to slowdown effect, that will be taken into consideration during further analysis.

\section{Conclusion}

As a result of this work the method for selecting the tritium nuclei with rigidity $0.5-3.0 \mathrm{GV}$ for PAMELA experiment data was developed and updated. It gave the possibility to separate the tritium nuclei by suppressing the background of hydrogen and helium isotopes. Counting of the number of tritium nuclei using this method allow to reconstruct the instrument spectrum, that will be done in nearest future.

\section{Acknowledgments}

This work was supported the Russian Ministry of Education and Science, project No 3.2131.2017. 


\section{References}

[1] Looper, M.D., Blake, J.B., Cummings, J.R. et al. SAMPEX Observations of Energetic Hydrogen Isotopes in the Inner Zone. Radiat. Meas. 1996;26 (6):967-978.

[2] Looper, M.D., Blake, J.B., Mewaldt, R.A. Maps of hydrogen isotopes at low altitudes in the inner zone from SAMPEX observations. Adv. Space Res. 1998;21(12):1679-1682.

[3] Bakaldin, A., Galper, A., Koldashov, S. et al. Geomagnetically trapped light isotopes observed with the detector NINA. J. Geophys. Res. Space Physics. 2002;107(A8):SMP 8-1-SMP 8-8.

[4] Bidoli, V., Casolino, M., De Pascale, M. et al. Isotope composition of secondary hydrogen and helium above the atmosphere measured by the instruments NINA and NINA-2. J. Geophys. Res. 2003;108:SMP 20-1-SMP 20-5

[5] Adriani, O., Barbarino, G. C., Bazilevskaya, G. A. et al. The PAMELA Mission: Heralding a new era in precision cosmic ray physics. Phys. Rep. 2014;544(4):323-370.

[6] Voronov, S.A., Danil'chenko. I.A., Koldobsky, S.A. A method for identifying deuterons in the PAMELA satellite-borne experiment. Instrum. Exp. Tech. 2011;54 (6):752-755. 